Z. Klin. Chem. Klin. Biochem.

13. Jg. 1975, S. 69-73

\title{
Empfindliche Mikromethode zur Bestimmung der UDP-Glucuronyltransferase-Aktivität in Leber- homogenat mit Bilirubin als Substrat ${ }^{1}$ )
}

\author{
Von W. Lehnert, Christa Wagner und W. Künzer \\ Univ.-Kinderklinik (Direktor: Prof. Dr. W. Künzer) Freiburg/Breisgau
}

(Eingegangen am 13. August/2. Dezember 1974)

Es wird eine zuverlässige Mikromethode zur Bestimmung der UDP-Glucuronyltransferase-Aktivität mit Bilirubin als Substrat beschrieben. Schon mit Leberhomogenaten, die $3 \mathrm{mg}$ Leber enthalten, lassen sich Bestimmungen mit einer Standardabweichung von $\pm 4,7 \%$ durchführen. Bei der Maus wurden mit dieser Methode Werte zwischen 85 und 300 und beim erwachsenen Menschen von etwa $240 \mathrm{nmol}$ Bilirubinglucuronid/40 $\mathrm{min} \times \mathrm{g}$ Leber gemessen.

\section{A sensitive microassay for UDP-glucuronyltransferase activity in liver homogenates with bilirubin as substrate}

A reliable and sensitive micromethod is described for the determination of UDP-glucuronyltransferase with bilirubin as substrate. Liver homogenates containing as little as $3 \mathrm{mg}$ of liver tissue are sufficient for the enzyme determination with a standard deviation of $\pm 4.7 \%$. Using this method values between 85 and 300 were found in mice, and about $240 \mathrm{nmol}$ bilirubinglucuronide/40 min $\times \mathrm{g}$ liver tissue, was found for the adult human.

1964 veröffentlichten Metge et al. (1) eine Mikromethode zur Bestimmung der UDP-Glucuronyltransferase-Aktivität (EC 2.4.1.17) in Leberbiopsieproben. Die Autoren inkubierten Leberhomogenat mit Bilirubin und UDP-Glucuronsäure, diazotierten den Inkubationsansatz mit ${ }^{35} \mathrm{~S}$-Sulfanilsäure, extrahierten die entstandenen radioaktiven Pigmente Azobilirubin bzw. Azobilirubinglucuronid $^{2}$ ) und trennten sie papierchromatographisch auf. Aus dem Radioaktivitätsverhältnis der beiden Azopigmente und der eingesetzten Menge an Bilirubin errechnet sich die Menge an gebildetem Bilirubinglucuronid, die der Aktivität des Enzyms proportional ist.

Leider konnten wir diese Methode trotz größter Anstrengungen nicht reproduzieren. Es scheint unmöglich, die beiden interessierenden Pigmente vom großen Überschuß an ${ }^{35} \mathrm{~S}$-Sulfanilsäure, ${ }^{35} \mathrm{~S}$-Diazobenzolsulfonsäure, der daraus entstehenden ${ }^{35} \mathrm{~S}-p$-Hydroxybenzolsulfonsäure und vielen anderen radioaktiven Verunreinigungen, die durch Diazotieren aromatischer Komponenten des Leberhomogenates entstehen, durch einfache Papierchromatographie abzutrennen.

1) Wesentliche Teile der vorliegenden Arbeit wurden von cand. med. Christa Wagner aus Göttingen als Dissertation den Medizinischen Fakultäten der Universität Freiburg i. Br. vorgelegt.

2) Der Einfachheit halber und aus historischen Gründen wird an der Bezeichnung der Pigmente mit „Azobilirubin" bzw. „Azobilirubinglucuronid“ festgehalten.
Andere Mikromethoden zur Bestimmung der UDPGlucuronyltransferase arbeiten mit unphysiologischen Substraten (2-7), mit nur schwer zugänglichem ${ }^{14} \mathrm{C}$ Bilirubin (8) oder benötigen zu große Lebermengen (9), als daß sie durch Leberblindpunktion an Neugeborenen gewonnen werden könnten.

Wir haben uns daher entschlossen, die vom Konzept her bestechende Methode von Metge et al. (1) so umzuarbeiten, daß sie ohne Schwierigkeiten zu reproduzieren sein sollte.

\section{Material und Methoden}

Geräte, Chemikalien, Lösungen

Potter-Elvehjem-Glashomogenisator, 2,5 ml Inhalt. Zentrifugengläschen, $15 \mathrm{ml}$, NS 14,5, mit spitzem Boden. Rotationsverdampfer mit „Spinne“, 6- und 12-armig (Büchi, Flavil, Schweiz). Flüssigszintillationszähler, Tricarb 3375 (Packard Instr., Frankfurt/M).

UDP-Glucuronsäure, Dinatriumsalz und Humanalbumin, reinst (Boehringer Mannheim). ${ }^{35} \mathrm{~S}$-Sulfanilsäure, $0,1 \%$ $(5,98 \mathrm{mmol} / 1)$ in $0,25 \mathrm{~mol} / 1 \mathrm{HCl}$, spez. Aktivität $50 \mathrm{mCi} / \mathrm{mmol}$ (Amersham-Buchler KG, Braunsch weig). Hyamine-Hydroxide $10-X, 1 \mathrm{~mol} / 1$ in Methanol (Packard Instr., Frankfurt/M). 2,2'-p-Phenylen-bis-(5-phenyloxazol) (POPOP), 2,5-Diphenyloxazol (PPO), Naph thalin für Szintillationszwecke und andere Chemikalien (Merck AG, Darmstadt). Cellulose-F-Dünnschichtplatten, $20 \times 20 \mathrm{~cm}$ (Merck AG, Darmstadt) ${ }^{3}$ ). MN-Cellulose-

3) Celluloseplatten ohne Fluoreszenzindikator zeigten andere Trenncigenschaften. 
pulver 300 für die Dünnschichtchromatographie und $\mathrm{MN}-\mathrm{Cel}$ lulosepulver für die Säulenchromatographie (Macherey Nagel, Düren). Absol. Äthanol 1.c. (7).

Inkubationslösung: $1,0 \mathrm{ml} 66 \mathrm{mmol} / 1$ Kaliumchlorid; $0,3 \mathrm{ml}$

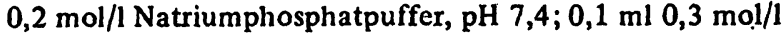
Magnesiumchlorid; $1,0 \mathrm{ml}$ isotone alkal ische Kaliumchloridlösung ( $154 \mathrm{mmol} / 1$ Kaliumchlorid und $0,32 \mathrm{mmol} / 1 \mathrm{Kalium}$ hydrogencarbonat). UDP-Glucuronsäurelösung: $0,3 \mathrm{mmol} / 1$ Dinatrium-UDP-Glucuronat.

Bilirubinlösung: $10 \mathrm{mg}$ reines Bilirubin werden so schnell wie möglich in $0,5 \mathrm{ml} 0,1 \mathrm{~mol} / 1$ Natriumhydroxid gelöst und mit bidest. Wasser auf $10 \mathrm{ml}$ aufgefüllt. $\mathrm{Zu} 0,5 \mathrm{ml}$ dieser Lösung gibt man sofort $0,5 \mathrm{ml}$ einer $50 \mathrm{~g} / 1$ Humanalbuminlösung und fullt mit bidest. Wasser auf $10 \mathrm{ml}$ auf. Die Lösung enthält $50 \mu \mathrm{g}=85,5 \mathrm{nmol}$ Bilirubin $/ \mathrm{ml}$. Sulfanilsäurelösung (nicht radioaktiv): $5 \mathrm{~g}$ Sulfanilsäure und $15 \mathrm{ml}$ konz. Salzsäure (etwa $12 \mathrm{~mol} / \mathrm{l}$ ) mit bidest. Wasser auf $1 \mathrm{l}$ auffüllen. Diazoreagenz: $100 \mathrm{ml}$ Sulfanilsäurelösung unmittelbar vor Gebrauch mit $5 \mathrm{ml}$ Natriumnitrit-Lösung $(72,5 \mathrm{mmol} / \mathrm{l})$ mischen.

${ }^{35} \mathrm{~S}$-Diazoreagenz: $\mathrm{Zu} 0,5 \mathrm{ml}$ der ${ }^{35} \mathrm{~S}$-Sulfanilsäurelösung gibt man kurz vor dem Gebrauch $25 \mu$ l Natriumnitrit-Lösung (72,5 $\mathrm{mmol} / \mathrm{l})$. Diotol: $65 \mathrm{mg}$ POPOP, 3,25 g PPO, 65,0 g Naphthalin, $250 \mathrm{ml}$ Toluol, $250 \mathrm{ml}$ Dioxan, $150 \mathrm{ml}$ Methanol.

Die UDP-Glucuronsäure-, Bilirubin- und Inkubationslösungen werden in etwa $2 \mathrm{ml}$-Portionen bei $-20^{\circ} \mathrm{C}$ längstens 2 Monate aufbewahrt.

Alle verwendeten organischen Lösungsmittel müssen unbedingt frisch destilliert sein.

\section{Durchführung der Versuche}

Einer in Ätheranästhesie durch Dekapitieren entbluteten Maus entnimmt man 20-40 mg Lebergewebe, wiegt auf einem Stückchen Parafilm genau aus und überfüht schnell in einen eisgekühlten Glashomogenisator, in den man pro $\mathrm{mg}$ Lebergewebe $20 \mu 1$ Inkubationslösung gibt. Es wird $3 \mathrm{~min}$ bei $300 \mathrm{U} / \mathrm{min}$ und $0^{\circ} \mathrm{C}$ homogenisiert.

Vom Homogenat werden $3 \mathrm{mg}$ Leber entsprechende Anteile $(63 \mu \mathrm{l})$ in vorgekühlte Zentrifugengläschen überführt, mit $20 \mu \mathrm{l}$ Bilirubin- (entsprechend 1,71 nmol Bilirubin) und $33 \mu \mathrm{l}$ UDP-Glucuronsäurelösung (entsprechend $10 \mathrm{nmol}$ UDP-Glucuronat) pro mg Leber versetzt und $40 \mathrm{~min}$ bei $37^{\circ}$ inkubiert. Die Zugabe der Bilirubinlösung muß präzise durchgeführt werden (Mikroliterspritze).

Man stoppt die Reaktion durch Kühlen mit Eiswasser und sofortige Zugabe von $0,5 \mathrm{ml}$ absol. Äthanol je Probe, diazotiert mit $100 \mu{ }^{35}$ S-Diazoreagenz und läßt $1 \mathrm{~h}$ bei $0^{\circ} \mathrm{C}$ im Kühlschrank stehen. Anschließend werden zu jeder Probe als Trägerund Markierungssubstanz $\mathbf{3 0} \mu \mathrm{l}$ eines aus Schweinegalle hergestellten Gemisches (s. u.) der beiden nicht radioaktiven Pigmente Azobilirubin und Azobilirubinglucuronid sowie je $4 \mathrm{ml}$ bidest. Wasser gegeben und der Farbstoff durch kräftiges Schütteln $(1 \mathrm{~min}) \mathrm{mit} 5 \mathrm{ml} n$-Butanol extrahiert. Nach Zentrifugieren (10 min, etwa $4500 \mathrm{U} / \mathrm{min},+4^{\circ} \mathrm{C}$ ) überführt $\operatorname{man} 4,5 \mathrm{ml}$ der organischen Phase in neue, vorgekühlte Zentrifugengläser, Schüttelt mit je $2 \mathrm{ml}$ bidest. Wasser $1 \mathrm{~min}$ aus, zentrifugiert (5 $\mathrm{min}$, etwa $4500 \mathrm{U} / \mathrm{min},+4^{\circ} \mathrm{C}$ ), pipettiert $3,5 \mathrm{ml}$ der organischen Phase in neue, vorgekühlte Zentrifugengläser und dampft das Lösungsmittel nach Zugabe von je $1 \mathrm{ml}$ bidest. Wasser und Vorkühlen der Gläschen in Eiswasser mit Hilfe einer Spinne am Rotationsverdampfer im Vakuum (etwa 1 Torr) bei $30^{\circ} \mathrm{C}$ zur Trockene ein. Man löst den Rückstand in je $200 \mu$ l Alkohol/ Wasser 1:1, benetzt alle farbstoffhaltigen Anteile der Glaswandungen, zentrifugiert kurz und dampft nach Vorkühlen der Gläschen in Eiswasser wiederum im Vakuum bei $30^{\circ} \mathrm{C}$ zur Trockene ein. Der Farbstoff wird in $30 \mu \mathrm{l}$ Essigsäure $(1 \mathrm{~mol} / \mathrm{l})$ aufgenommen und in etwa $10 \mu \mathrm{l}$-Portionen auf Cellulose-F-Dünnschichtplatten so aufgetragen, daß sich der Startpunkt je 2,5 $\mathrm{cm}$ vom linken und unteren Rand der Platte befindet. Zwischendurch wird mit einem kalten Luftstrom getrocknet. Zum Schluß tropft man mit einer Mikroliterspritze so viel verdünnte (1 mol/l) Essigsäure (etwa 10-15 $\mu \mathrm{l}$ ) langsam auf die Mitte des Farbflecks, bis dieser über seinen Rand hinaus benetzt ist. Man erreicht dadurch eine gleichmäßige Adsorption der Pigmente an der Cellulose und im Gefolge davon eine tailing-freie Chromatographie. Es wird anschließend $10 \mathrm{~min}$ unter einem kalten Luftstrom getrocknet.

Die Chromatographie erfolgt zweidimensional. Durch zwei Reinigungsläufe in der ersten Richtung mit alsol. Äthanol (45 $\mathrm{min}$ ) bzw. verdünnte Essigsäure $(0,17 \mathrm{~mol} / 1)(90 \mathrm{~min})$ werden die interessierenden Azopigmente von fast allen radioaktiven Verunreinigungen befreit und dann durch Chromatographie in der zweiten Richtung mit Methyläthylketon/Aceton/ Propionsäure/Wasser (Volumina $55 \mathrm{ml}+20 \mathrm{ml}+25 \mathrm{ml}+35 \mathrm{ml}$ ) als Laufmittel aufgetrennt $(3 \mathrm{~h})$. Nach jedem Lauf müssen die Platten 20 min unter Föhnen mit kalter Luft getrocknet werden.

Zur Auswertung zeichnet man um die beiden Farbflecke (RFAzobilirubin: etwa 0,68, $\mathbf{R}_{\text {FAzobilirubinglucuronid: etwa }}$ 0,53 ) mit Bleistift gleich große Vierecke (Höhe 1,8 cm; Breite, je nach Größe des Farbfleckes, meist $2 \mathrm{~cm}$ ) und überführt die die Pigmente enthaltende Cellulose nach Abkratzen mit einem Metallspatel mit Hilfe von Glanzpapier in Zählgläschen. Zwischen dem Azobilirubinglucuronidfleck und dem Auftragspunkt, jedoch mindestens $1,6 \mathrm{~cm}$ von letzterem entfernt, entnimmt man einen Leerwert gleicher Flächengröße und versetzt alle Proben nacheinander mit je 0,1 ml Eisessig (schütteln), $1,0 \mathrm{ml}$ Hyamin-Hydroxyd (schütteln) und $10 \mathrm{ml}$ Diatol (schütteln) und mißt die Radioaktivität im Flüssigszintillationszähler.

Die gemessene Radioaktivität des Leerwertes wird von der des Azobilirubinglucuronidflecks abgezogen.

Die Menge an gebildetem Glucuronid errechnet sich wie folgt:

$\mathrm{BG}[\mathrm{nmol} / 40 \mathrm{~min} \times \mathrm{g}$ Leber $]=$

$\frac{\text { Akt }_{\text {ABG }}[\mathrm{Imp} / \mathrm{min}]-\mathrm{Akt}_{\mathrm{LW}}[\mathrm{Imp} / \mathrm{min}]}{\mathrm{Akt}_{\text {gesamt }_{\text {f }}[\mathrm{Imp} / \mathrm{min}]}} \times \mathbf{B}[\mathrm{nmol} / \mathrm{g}$ Leber $]$

BG = gebildete Bilirubinglucuronidmenge

Akt $_{\mathrm{ABG}}=$ Radioaktivität des Azobilirubinglucuronidfleckes

$\mathrm{Akt}_{\mathrm{LW}}=$ Radioaktivität des Leerwertes

$\mathrm{Akt}_{\text {gesamt }}=\mathrm{Akt}_{\mathrm{AB}}+\mathrm{Akt}_{\mathrm{ABG}}-\mathrm{Akt}_{\mathrm{LW}}$

$\mathrm{Akt}_{\mathrm{AB}}=$ Radioaktivität des Azobilirubinfleckes

$\mathrm{B}$ = zugegebene Bilirubinmenge

Darstellung eines Gemisches aus Azobilirubin und Ażobilirubinglucuronid aus Schweinegalle

Man verdünnt $300 \mathrm{ml}$ frischer Schweinegalle mit $300 \mathrm{ml}$ bidest. Wasser, gibt $300 \mathrm{ml}$ absol. Alkohol hinžu, bringt unter Rühren mit Eisessig auf pH 4, kühlt auf $0^{\circ} \mathrm{C}$ ab und diazotiert mit 315 ml eiskaltem Diazoreagenz unter Rühren. Nach $15 \mathrm{~min}$ gibt man nochmals $300 \mathrm{ml}$ Alkohol und $\mathbf{3 1 5} \mathrm{ml}$ Diazoreagenz hinzu und rührt $1 \mathrm{~h}$ bei $0^{\circ} \mathrm{C}$. Dann wird wie folgt extrahiert:

Je $1 \times$ mit 600 bzw. $300 \mathrm{ml}$ Petroläther $\left(40-60^{\circ} \mathrm{C}\right)$

$1 \times$ mit $270 \mathrm{ml}$ Petroläther $\left(40 \div 60^{\circ} \mathrm{C}\right) / \mathrm{n}$-Butanol (Volumina $70 \mathrm{ml}+20 \mathrm{ml})$

$2 \times$ mit $300 \mathrm{ml}$ Petroläther $\left(40-60^{\circ} \mathrm{C}\right) / \mathrm{n}$-Butanol (Volumina $70 \mathrm{ml}+30 \mathrm{ml}$ )

$1 \times$ mit $450 \mathrm{ml}$ Chloroform und

$4 \times$ mit $300 \mathrm{ml}$ Chloroform

Die wäßrige Phase, in der sich die interessierenden Pigmente befinden, dampft man im Vakuum (etwa 1 Torr) am Rotationsverdampfer bei $30^{\circ} \mathrm{C}$ zur Trockene ein, nimmt den Rückstand in $450 \mathrm{ml}$ verdünnter Essigsäure $(0,17 \mathrm{~mol} / 1)$ auf, verrührt mit $150 \mathrm{~g}$ MN-Cellulose 300 für die Dünnschichtchromatographie und nutscht ab. Der Filterrückstand wird nochmals mit $300 \mathrm{ml}$ verdünnter Essigsäure $(0,17 \mathrm{~mol} / \mathrm{l})$ verrührt und wiederrum $\mathrm{ab}$ genutscht. Man extrahiert den an die Cellulose adsörbierten Farbstoff $3 \mathrm{mal} \mathrm{mit} \mathrm{je} 375 \mathrm{ml}$ Alkohol/Aceton/Wasser (Volumina $100 \mathrm{ml}+100 \mathrm{ml}+100 \mathrm{ml}$ ) und dampft die vereinigten Farbstofflösungen im Wasserstrahlvakuum bei $30^{\circ} \mathrm{C}$ zur Trockene ein. Zur weiteren Reinigung werden die Pigmente, in $10-15 \mathrm{ml}$ verd. Essigsäure $(0,17 \mathrm{~mol} / 1)$ gelöst, $2 \mathrm{mal}$ an MN-Cellulose für die Säulenchromatographịie chromatographiert. 
$\mathrm{Zu}$ diesem Zweck gießt man eine Säule $(3,7 \mathrm{~cm} \emptyset)$ aus $150 \mathrm{~g}$ Cellulose und $900 \mathrm{ml}$ verd. Essigsäure $(0,17 \mathrm{~mol} / \mathrm{l})$, läßt zur Konditionierung der Säule mit dem gleichen Elutionsmittel über Nacht laufen und trägt dann erst die Farbstofflösung auf. Eluiert wird so lange mit verd. Essigsäure $(0,17 \mathrm{~mol} / 1)$, bis sich die beiden Farbstoffe, nach Elution verschiedener, auch violett gefärbter Anteile und einer gelblichen Zone, in zwei breite Banden aufgetrennt haben, von denen die schneller wandernde das Azobilirubinglucuronid enthält. Man schneidet die beiden Zonen heraus, extrahiert die Farbstoffe $3 \mathrm{mal} \mathrm{mit} \mathrm{je} 100 \mathrm{ml}$ Alkohol/Aceton/Wasser (Volumina $100 \mathrm{ml}+100 \mathrm{ml}+100 \mathrm{ml}$ ), dampft die Extrakte im Vakuum bei $30^{\circ} \mathrm{C}$ zur Trockene ein und rechromatographiert nach Auflösen in etwa $15 \mathrm{ml}$ verd. Essigsäure $(0,17 \mathrm{~mol} / 1)$ wie beschrieben.

Die beiden Pigmente werden nun getrennt in je 1-2 ml bidest. Wasser aufgenommen und zur weiteren Reinigung an CelluloseF-Dünnschichtplatten chromatographiert. Pro Platte trägt man etwa $0,3 \mathrm{ml}$ Farbstofflösung als Strich im Abstand von $3 \mathrm{~cm}$ vom unteren Rand auf und tropft, um die Pigmente gleichmäßig auf der Celluloseschicht zu verteilen, nach kurzem Zwischentrocknen verd. Essigsäure $(0,17 \mathrm{~mol} / \mathrm{l})$ auf die Auftragszone. Chromatographiert wird, nach Trocknen im kaltey/ Luftstrom, $2 \mathrm{~h}$ mit verd. Essigsäure $(0,17 \mathrm{~mol} / \mathrm{l})$ als Laufmittel und nach Zwischentrocknen der Platten (10 min), mit Methyläthylketon/Aceton/Propionsäure/Wasser (Volumina $55 \mathrm{ml}+$ $20 \mathrm{ml}+35 \mathrm{ml}+25 \mathrm{ml})(3 \mathrm{~h})$ in der gleichen Richtung! Nach Beendigung der Chromatographie trocknet man kurz, kratzt die interessierenden Zonen aus, extrahiert die Farbstoffe mit 20-30 ml Alkohol/Aceton/Wasser (Volumina, $100 \mathrm{ml}+100 \mathrm{ml}$ $+100 \mathrm{ml}$ ) und dampft die Extrakte im Vakuum bei $30^{\circ} \mathrm{C}$ zur Trockene ein. Die Rückstände werden in 1-2 ml bidest. Wasser aufgenommen. Man mischt die Pigmente nun so, daß beide in etwa gleicher Konzentration vorliegen (Probechromatogramm).

Die Farbstofflösung wird bei $+4^{\circ} \mathrm{C}$ im Eisschrank aufbewahrt und ist mindestens 3-6 Monate haltbar.

Der Wichtigkeit halber sei nochmals darauf hingewiesen, daß

1. alle Lösungsmittel frisch destilliert und

2. alle Glasgeräte peinlichst sauber sein müssen (Chromschwefelsäure).

Dies gilt für den gesamten Versuchsteil.

\section{Ergebnisse}

\section{Optimierung der Versuchsbedingungen}

Einfluß der Magnesiumionenkonzentration auf die Konjugation

Etwa $40 \mathrm{mg}$ Leber wurden mit $20 \mu \mathrm{l}$ Inkubationslösung (ohne Magnesiumionen)/mg Leber homogenisiert. Wir entnahmen $4 \mathrm{mal} 2$ Proben von je $3 \mathrm{mg}$ Leber, fügten $0,0,125,0,25$ bzw. 0,5 $\mu \mathrm{mol}$ Magnesiumchlorid, je $10 \mu \mathrm{l}(0,855 \mathrm{nmol})$ Bilirubin- und 16,6 $\mu \mathrm{l}(5 \mathrm{nmol})$ UDP-Glucuronsäurelösung pro mg Leber hinzu und arbeiteten wie beschrieben weiter. Es zeigte sich, daß alle verwendeten Magnesiumionenkonzentrationen die Ausbeute an Glucuronid gegenüber den Proben ohne Magnesiumionen $(100 \%)$ auf etwa $220 \%$ steigerten. Für die weiteren Versuche wurde eine Magnesium-Konzentration von $0,25 \mu \mathrm{mol} / \mathrm{mg}$ Leber eingesetzt.

\section{Abhängigkeit der Konjugation von der Bilirubinmenge}

$\mathrm{Zu}$ je 6 Doppelproben Leberhomogenat (3 mg Leber/ Probe) wurden bei überschüssiger UDP-Glucuronsäure (400 nmol/mg Leber) steigende Mengen an Bilirubin hinzugegeben und wie beschrieben weitergearbeitet.

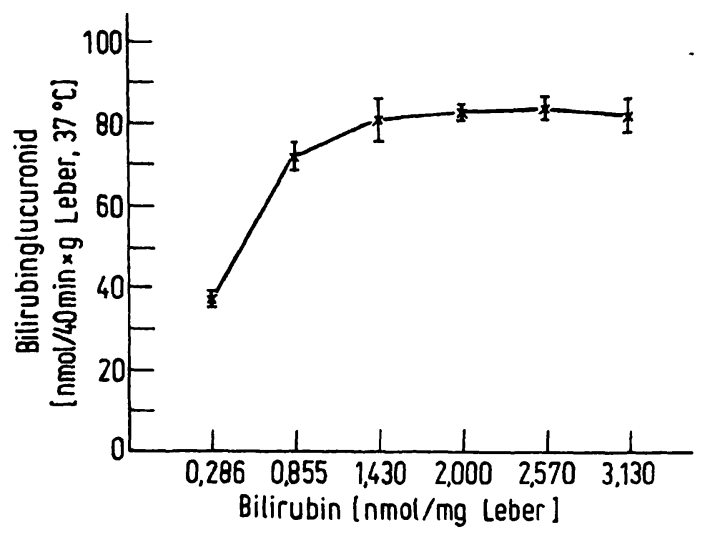

Abb. 1. Abhängigkeit der Konjugation von der Bilirubinkonzentration.

Inkubationsansatz: $3 \mathrm{mg}$ Leber in $60 \mu 1$ Inkubationslösung; $400 \mathrm{nmol}$ UDP-Glucuronsäure/mg Leber und steigende Mengen Bilirubin. Die für die Durchführung der Bestimmung empfohlene Menge von 1,71 nmol Bilirubin/mg Leber liegt im Sättigungsbereich der Kurve.

Wie Abbildung 1 zu entnehmen ist, befindet sich der Arbeitspunkt mit 1,71 nmol Bilirubin/mg Leber im Sättigungsbereich.

\section{Abhängigkeit der Konjugation von der UDP-Glucuron- säuremenge}

Da sich ein exzessiver Überschuß an Bilirubin aus technischen Gründen verbietet (Schwierigkeiten bei der Chromatographie und Auswertung), arbeiteten wir mit 2,85 nmol Bilirubin/mg Leber. Mit steigenden Mengen an UDP-Glucuronsäure im Ansatz erhält man die in Abbildung 2 wiedergegebene Sättigungskurve, der zu entnehmen ist, daß $10 \mathrm{nmol}$ UDP-Glucuronsäure/mg Leber ausreichen.

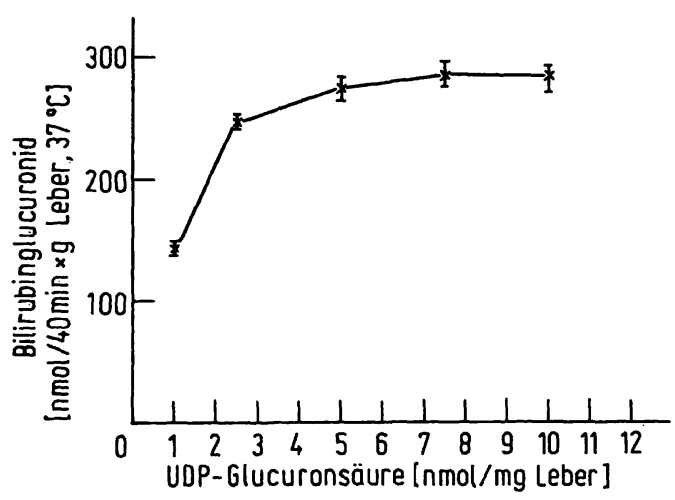

Abb. 2. Abhängigkeit der Konjugation von der UDP-Glucuronsäurekonzentration.

Inkubationsansatz: $3 \mathrm{mg}$ Leber in $60 \mu \mathrm{l}$ Inkubationslösung; $2,85 \mathrm{nmol}$ Bilirubin/mg Leber und steigende Mengen UDP-Glucuronsäure. Die für die Durchführung der Bestimmung empfohlene Menge an UDP-Glucuronsäure (10 $\mathrm{nmol} / \mathrm{mg}$ Leber) liegt im Sättigungsbereich der Kurve. 


\section{Abhängigkeit der Konjugation von der Homogenisie- rungsdauer}

Etwa $40 \mathrm{mg}$ Leber wurden mit $20 \mu \mathrm{l}$ Inkubationslösung/ mg Leber bei $0^{\circ} \mathrm{C}$ und $300 \mathrm{U} / \mathrm{min}$ homogenisiert: Wir entnahmen nach verschiedenen Zeiten je 2 Proben (a $3 \mathrm{mg}$ Leber), stellten sie in Eiswasser und arbeiteten dann alle zusammen wie beschrieben auf. Der Versuch zeigt, daß die Enzymaktivität von der Homogenisierungsdauer weitgehend unabhängig ist. Wir haben daher die anfangs gewählte Homogenisierungsdauer von $3 \mathrm{~min}$ bei $300 \mathrm{U} / \mathrm{min}$ beibehalten.

\section{Variation der Inkubationszeit}

6 Doppelproben (3 mg Leber/Probe) wurden wie oben beschrieben behandelt und verschieden lange inkubiert. Abbildung 3 ist zu entnehmen, daß keine lineare Beziehung zwischen Konjugation und Inkubationszeit besteht. Die maximale Ausbeute an Glucuronid wird bei einer Inkubationsdauer von etwa 40 min erreicht.

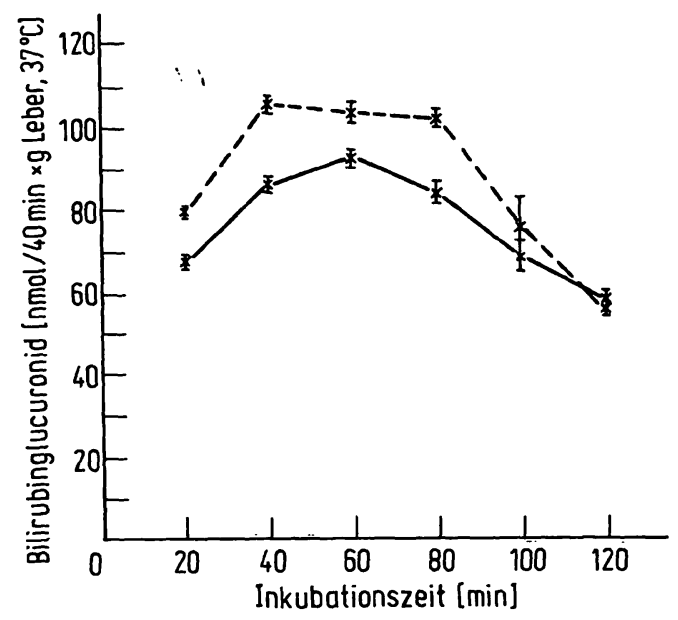

Abb. 3. Abhängigkeit der Konjugation von der Inkubationszeit. Inkubationsansatz: $3 \mathrm{mg}$ Leber in $60 \mu 1$ Inkubationslösung; $1,71 \mathrm{nmol}$ Bilirubin und $10 \mathrm{nmol}$ UDP-Glucuronsäure/mg Leber. Im Bereich zwischen 20 und 120 min Inkubationsdauer besteht $z$ wischen der Konjugation und der Inkubationszeit keine lineare Beziehung.

\section{Versuche zur Reproduzierbarkeit}

In einem ersten Versuch wurden 6 identische Proben (etwa $3 \mathrm{mg}$ Leber) eines Homogenates wie üblich aufgearbeitet. Die Werte lagen zwischen 198,5 und 207,8 nmol Bilirubinglucuronid/g Leber $\times 40$ min mit einer Näherungsstandardabweichung (10) von $\pm 2,13$. Um zu überprüfen, inwieweit die Genauigkeit der Methode von der Größe der verwendeten Gewebsprobe und von den Manipulationen bis einschließlich dem Homogenisieren abhängig ist, wurden 3 verschiedenen Mäusen je 4 benachbarte Leberstücke (Zwischen 27,6-42,6, 15,821,5 bzw. 5,6-12,5 mg) entnommen, sofort bei $-78^{\circ} \mathrm{C}$ eingefroren und nacheinander unter identischen
Bedingungen verarbeitet (Doppelbestimmungen). Die Mittelwerte für die drei Gewichtsgruppen liegen bei 176,5, 151,2 bzw. 138,6 nmol Bilirubinglucuronid/g Leber $\times 40 \mathrm{~min}$ bei Näherungsstandardabweichungen von $\pm 5,8,4,21$ bzw. 4,74. Die nach logarithmischer Transformation aus 96 Doppelbestimmungen errechnete Standardabweichung beträgt. $\pm 4,7 \%{ }^{4}$ ).

\section{Untersuchungen zum Verhalten dér Enzym-} aktivität

Es wurde geprüft, ob sich die Enzymaktivität beim Stehen des Homogenates bei $0^{\circ} \mathrm{C}$ verändert. Bestimmungen an Aliquoten einer größeren Probe nach 0, 20, 40, 60 und 80 min ergaben identische Werte. Bewahrt man die Leberprobe im verschlossenen Glasröhrchen 5 Tage bei $-78^{\circ} \mathrm{C}$ auf, so sinkt die gemessene Enzymaktivität auf etwa $63 \%$ des ursprünglichen Wertes.

\section{Hydrolaseaktivität im Leberhomogenat}

Nach der angegebenen Methode wurde ${ }^{35}$ S-Azobilirubinglucuronid dargestellt (zehnfacher Ansatz) und mit je zwei Leberhomogenatproben mit bzw. ohne Saccharo1.4-lacton ( $\beta$-Glucuronidasehemmer) im Ansatz inkubiert. Nach $40 \mathrm{~min}$ bei $37^{\circ} \mathrm{C}$ waren in beiden Versuchen etwa $30 \%$ des eingesetzten Glucuronides zerfallen.

\section{Diskussion}

Es bestehen heute kaum mehr Zweifel darüber, daß in der Leberzelle mehrere UDP-Glucuronyltransferasen existieren $(11,12)$, deren Beziehungen untereinander jedoch noch weitgehend unbekannt sind. Daher empfiehlt es sich, für Untersuchungen, die über die Fähig: keit der Leber, Bilirubin zu konjugieren Auskunft geben sollen, auch eine Ënzymbestimmungsmethode zu wählen, die mit Bilirubin als Substrat arbeitet.

Die photometrische Auswertung der Van-den-BerghReaktion, die diesen Methoden meist zugrunde liegt, · stößt bei kleinen und kleinsten Enzymmengen bald auf unüberwindliche Schwierigkeiten. Eine Alternative bietet nur schwer zugängliches radioaktives natürliches (8) bzw. synthetisches (13) Bilirubin oder, was weit einfacher ist, die nachträgliche radioaktive Markierung durch Diazotieren mit radioaktiven Diazoniumverbindungen, wie sie schon von Metge et al. (1) durchgeführt wurde.

Da wir jedoch, wie schon eingangs kurż erwähnt, letżtere im Konzept interessante. Methode trotz besten Bemühens nicht reproduzieren konnten, haben wir uns zu ihrer Uberarbeitung entschlossen.

${ }^{4}$ ) Herrn Dr. H. Jesdinsky vom Institut für Medizinische Statistik und Dokumentation der Universität Freiburg danken wir für die Berechnung der Standardabweichung. 
Die schwächste Stelle dieser Methode stellt ohne Zweifel die chromatographische Trennung der Diazopigmente voneinander und von anderen radioaktiven Produkten dar. Wir haben diesem wichtigen Schritt daher erhöhte Aufmerksamkeit geschenkt.

Die Einführung der zweidimensionalen Dünnschichtchromatographie mit drei verschiedenen Lösungsmitteln erbrachte eine einwandfreie, autoradiographisch überprüfte Trennung.

Besondere Beachtung fand die Präparation und Reinigung der nichtradioaktiven Azopigmente, denn wir konnten feststellen, daß unreine Präparate die Komplexizität des zu trennenden radioaktiven Gemisches erhöhten. Dies wäre dadurch zu erklären, daß im unreinen Azopigmentpräparat Substanzen vorhanden sind, die mit dem im Überschuß vorliegenden radioaktiven Diazoreagenz reagieren.

Zur Optimierung der Versuchsbedingungen wurden verschiedene Parameter variiert und die Ergebnisse, falls nötig, bei den nachfolgenden Versuchen berücksichtigt.

Wie zu erwarten, konnte die Glucuronidbildung durch Zugabe von Magnesiumionen um etwa $120 \%$ gesteigert werden. Bisher wurde dieser Effekt durch die Aktivierung der Glucuronyltransferase erklärt $(9,14,15)$. Zakim et al. (12) konnten jedoch durch kinetische Untersuchungen zeigen, daß Magnesiumionen auf die Aktivität des Enzyms keinen Einfluß haben, sondern daß es sich bei dem beobachteten Effekt um eine Steigerung der Löslichkeit des freien Bilirubins handelt, das meist an Albumin gebunden eingesetzt wird. Nur das freie Bilirubin, dessen Löslichkeit bei physiologischen pH-Werten sehr gering ist, wird von der UDP-Glucuronyltransferase als Substrat umgesetzt.

Bei Variation der Bilinubinkonzentration in Gegenwart überschüssiger UDP-G̣lụcuronșạure ergibt sich die in
Abbildung 1 dargestellte Sättigungskurve, die zeigt, daß sich der Arbeitspunkt mit 1,71 nmol Bilirubin/mg Leber im Sättigungsbereich befindet.

Die Durchführung des reziproken Versuches war nicht ohne weiteres möglich. Bei zu großen Bilirubinmengen im Ansatz wird das Verhältnis der beiden radioaktiven Azopigmente zu ungünstig, weil dann das bei der Dünnschichtchromatographie schneller wandernde Azobilirubin eine zu kräftige radioaktive Spur hinterläßt, aus der sich die Radioaktivität des Azobilirubinglucuronids nicht mehr ausreichend hervorhebt. Mit 2,85 nmol Bilirubin/mg Leber, einer Konzentration, wie sie auch Menken et al. (8) für ihre Bestimmungsmethode verwendeten, erhält man die in Abbildung 2 wiedergegebene Kurve, der zu entnehmen ist, daß die verwendete UDPGlucuronsäurekonzentration von $10 \mathrm{nmol} / \mathrm{mg}$ Leber im Sättigungsbereich liegt.

Im Gegensatz zur Bestimmung der UDP-Glucuronyltransferase-Aktivität mit $p$-Nitrophenol als Substrat (7), hat die Homogenisierungsdauer im Bereich zwischen 0,5 und 5,0 min auf die Enzymaktivität keinen Einfluß.

Bei Variation der Inkubationszeit ergibt sich die in Abbildung 3 dargestellte Abhängigkeit der Glucuronidsynthese, die im untersuchten Bereich nicht linear ist. Die gemessenen Werte dürfen daher nicht auf beliebige Inkubationszeiten extrapoliert werden. Eine differenzierte Beurteilung der Meßwerte sollte anhand von zu erstellenden Normalwerten vorgenommen werden. Die Nichtlinearität kann Ausdruck der gefundenen unspezifischen Hydrolaseaktivität im Homogenat sein, die wir auch mit $p$-Nitrophenylglucuronid nachweisen konnten (7). Die mit dieser Methode gemessenen UDPGlucuronyltransferase-Aktivitäten lagen beim Erwachsenen um $240 \mathrm{nmol}$ Bilirubinglucuronid/40 $\mathrm{min} \times \mathrm{g}$ Leber.

\section{Literatur}

1. Metge, W. R., Owen, C. A., Foulk, W. T. \& Hoffman, H. N. (1964), J. Lab. Clin. Med. 64, 335-341

2. Frei, J., Schmid, E. \& Birchmeier, H. (1970), in Bergmeyer, H. U. "Methoden der enzymatischen Analyse", Bd. II, S. 679, Verlag Chemie, Weinheim/Bergstr.

3. Wong, K. P. (1969), Clin. Chim. Acta 26, 119-126

4. Cotte, J., Mathieu, M., Andre, J. P., Collombel, C. \& Padis, L. (1967), Enzy mol. Biol. Clin. 8, 387.

5. Temple, A. R., Done, A. K. \& Clement, M. S. (1971), J. Lab. Clin. Med. 77, 1015-1019.

6. Marniemi, J. \& Hänninen, O. (1973), FEBS Lett. 32, 273.

7. Lehnert, W., Limberg, J. \& Künzer, W. (1974), diese Z., 12, 23-27.

8. Menken, M., Barret, P. V. D. \& Berlin, N. I. (1966), Clin. Chim. Acta 14, 777-785.

9. Van Roy, F. P. \& Heirwegh, K. P. M. (1968), Biochem. J. 107, 507-518. Black, M., Billing, B. H. \& Heirwegh, K. P. M. (1970), Clin. Chim. Acta 29, 27-35.
10. Kaiser, R. (1965), „Chromatographie in der Gasphase“, Bd. IV, S. 32, Hochschultaschenbücher-Verlag, Mannheim.

11. Howland, R. D., Burkhalter, A., Trevor, A. J., Hegeman, S. \& Shirachi, D. Y. (1971), Biochem. J. 125, 991-997.

12. Zakim, D., Goldberg, J. \& Vessey, D. A. (1973), Biochim. Biophys. Acta 309, 67-74.

Vessey, D. A., Goldberg, J. \& Zakim, D. (1973), Biochim. Biophys. Acta 309, 75-82.

13. Plieninger, H., El-Barkawi, F., Ehl, K., Kohler, R. \& McDonagh, A. F. (1972), Liebigs Ann. Chem. 758, 195201.

14. Lathe, G. M. \& Walker, M. (1958), Biochem. J. 70, 705-709.

15. Wong, K. P. (1971), Biochem. J. 125, 27-35
Willy Lehnert Univ.-Kinderklinik 78 Freiburg Mathildenstr. 1 
